University of Wollongong

Research Online

Faculty of Engineering and Information

Faculty of Engineering and Information

Sciences - Papers: Part A

Sciences

$1-1-2013$

\title{
Involvement of consumers in health technology assessment activities by inahta agencies
}

David Hailey

University of Wollongong, dhailey@uow.edu.au

Sophie Werko

Swedish Council on Health Technology Assessment

Rugayah Bakri

Ministry of Health Malaysia

Alun Cameron

Australian Safety and Efficacy Register of New Interventional Procedures -Surgical

Britta Gohlen

German Institute for Medical Documentation and Information

See next page for additional authors

Follow this and additional works at: https://ro.uow.edu.au/eispapers

Part of the Engineering Commons, and the Science and Technology Studies Commons

Research Online is the open access institutional repository for the University of Wollongong. For further information contact the UOW Library: research-pubs@uow.edu.au 


\title{
Involvement of consumers in health technology assessment activities by inahta agencies
}

\begin{abstract}
Objectives: To obtain further information from members of the International Network of Agencies for Health Technology Assessment (INAHTA) on the involvement of consumers in their programs. Methods: A questionnaire for a survey was developed and sent to member agencies in November 2010. Survey responses were compared with those from an earlier survey conducted in 2005. Results: Of the thirtythree agencies that provided responses, 67 percent involve consumers in some aspects of their health technology assessment (HTA) programs, compared with 57 percent in 2005. As in the earlier survey, most agencies reporting involvement have contact with consumer or patient organizations and a large minority also involve individual consumers. Summaries of HTA reports that are intended to be easily understood by consumers are prepared by 84 percent of the agencies, and 42 percent involve consumers in dissemination of HTA material. In both areas, there was some increase from the levels previously reported. Conclusions: The survey results suggest that there is a trend to increased involvement of consumers by the INAHTA agencies in their programs but that the level of involvement remains relatively limited. The manner of consumer participation varies between agencies. (C) Cambridge University Press 2012.
\end{abstract}

\section{Keywords}

involvement, technology, consumers, assessment, activities, inahta, agencies, health

\author{
Disciplines \\ Engineering | Science and Technology Studies
}

\section{Publication Details}

Hailey, D., Werko, S., Bakri, R., Cameron, A., Gohlen, B., Myles, S., Pwu, J. \& Yothasamut, J. (2013). Involvement of consumers in health technology assessment activities by inahta agencies. International Journal of Technology Assessment in Health Care, 29 (1), 79-83.

\section{Authors}

David Hailey, Sophie Werko, Rugayah Bakri, Alun Cameron, Britta Gohlen, Susan Myles, Jasmine Pwu, and Jomkwan Yothasamut 


\section{INVOLVEMENT OF CONSUMERS IN HEALTH TECHNOLOGY ASSESSMENT ACTIVITIES BY INAHTA AGENCIES}

David Hailey

University of Wollongong, Australia

Sophie Werkö

Swedish Council on Health Technology Assessment

Rugayah Bakri

Ministry of Health Malaysia

Alun Cameron

Australian Safety and Efficacy Register of New Interventional Procedures -Surgical
Britta Göhlen

German Institute for Medical Documentation and Information

Susan Myles

Healthcare Improvement Scotland

Jasmine Pwu

Center for Drug Evaluation

Jomkwan Yothasamut

Health Intervention and Technology Assessment Program

For the INAHTA Working Group on Impact of HTA

Objectives: To obtain further information from members of the International Network of Agencies for Health Technology Assessment (INAHTA) on the involvement of consumers in their programs. Methods: A questionnaire for a survey was developed and sent to member agencies in November 2010. Survey responses were compared with those from an earlier survey conducted in 2005. Results: Of the thirty-three agencies that provided responses, 67 percent involve consumers in some aspects of their health technology assessment (HTA) programs, compared with 57 percent in 2005. As in the earlier survey, most agencies reporting involvement have contact with consumer or patient organizations and a large minority also involve individual consumers. Summaries of HTA reports that are intended to be easily understood by consumers are prepared by 84 percent of the agencies, and 42 percent involve consumers in dissemination of HTA material. In both areas, there was some increase from the levels previously reported.

Conclusions: The survey results suggest that there is a trend to increased involvement of consumers by the INAHTA agencies in their programs but that the level of involvement remains relatively limited. The manner of consumer participation varies between agencies.

Keywords: Health Technology assessment, Biomedical, Agencies, Patients, Consumer participation

The focus of health technology assessment (HTA) has often been on stakeholders with major financial or operational responsibilities for health technologies, including government authorities, manufacturing industry, healthcare professionals, and the health insurance industry. Patients and their families and carers are also a key group with major interests in health technologies, but until quite recently limited attention has been given to involving them in the HTA process (1). Criticism of the situation included comment that health experts such as physicians, researchers, and economists largely dominate HTA decision making. This was seen as antithetical to the belief that acceptable health decision making requires a process that is transparent, not dominated by any particular interest and reflects the values of all users (2).

In 2005 the International Network of Agencies for Health Technology Assessment (INAHTA) prepared a review to help consideration of how it might encourage more extensive involvement of patients in HTA (1). Involvement of patients in formulation of questions, in assessments and in dissemination had received some consideration in the literature, although there was little that specifically related to the situation typically facing
HTA agencies of routinely producing and disseminating advice for decision makers in health care, often with tight deadlines. Some of the literature on involving consumers with health research provided some issues to consider (3-6). Common themes included interaction of consumers and researchers, resources, technical demands on consumers, training and education, nature and extent of consumer representation, matching consumer information and information from the literature, time demands and remuneration, and consumer impact on discussion and decisions.

One of the earlier reports of consumer involvement in HTA gave details of approaches used in England by the National Coordinating Centre for Health Technology Assessment (NCCHTA). These included attending prioritization meetings, and commenting on research need for particular topics, research proposals, and research reports (7).

Later that year members of INAHTA were surveyed to obtain information on their involvement with consumers. The term "consumers" was taken to include patients, carers, longterm users of services, organizations representing consumers' interests, and members of the public, following a definition 
developed by the English HTA program (7). The survey found that most INAHTA members interacted with consumers in some aspects of their programs, although not always routinely. Involvement seemed likely to increase in the future (8).

These activities of INAHTA overlapped with the creation of the Health Technology international Interest Sub-Group (ISG) for Patient and Citizen Involvement in HTA. The ISG has since developed as a strong forum for promotion of processes for incorporating patients' perspectives into HTAs and engaging patients in the HTA process (9). There has been continued contact between INAHTA and the ISG.

Some more recent literature has further explored issues and practicalities associated with the engagement of patients and the community in the HTA process. Lee et al. have drawn attention to issues with HTA reports that included patient-related assessments (10). There was considerable variation in how comprehensively the issues were managed and in the methodological transparency of the reports that they reviewed, giving uncertainty as to their relevance and validity. Gagnon et al., in a systematic review, conclude there are few published examples of experiences of involving patients and the public in HTA (11). They see a need to develop more systematic approaches to considering patients' and the public's perspectives in HTA.

Recent articles suggest there is some progress in the use by HTA agencies of input from patients or the community but that further evaluation of process and effectiveness is needed $(12 ; 13)$. From a study based on interviews with people in HTA agencies, Gauvin et al. found that the HTA community is moving toward greater public involvement but remains cautious and ambivalent about the technical feasibility of public involvement, acceptability to policy makers and practitioners, and its impact on agencies' resources and procedures. They suggest there is a need for rigorous evaluations to inform HTA agencies' decisions on adoption or rejection of public involvement practices (12).

In 2010, a follow-up survey was undertaken by INAHTA to obtain information on whether its member agencies had changed their approaches in involving consumers since the earlier survey. This initiative reflected the increasing interest that has been expressed in gathering evidence about patients' perspectives, and in ensuring their engagement in the HTA process (9). In this article, we present the main findings from the survey and a comparison with the earlier results.

\section{METHODS}

The questions and the definition of consumers used in the earlier survey were considered to still be appropriate and were used again for the follow up. The opportunity was taken to liaise with the HTAi ISG on Patient/Citizen Involvement in HTA on details of the survey. Following suggestions by the ISG, two items were added to the survey questionnaire, covering the inclusion of consumer perspectives in assessments.
Table 1. Distribution of Agencies Providing Responses, by Region

\begin{tabular}{lcc}
\hline & $2010, \%$ & $2005, \%$ \\
\hline Europe & 67 & 68 \\
Asia & 15 & 3 \\
Australasia & 6 & 8 \\
North America & 6 & 16 \\
Latin America & 6 & 5 \\
\hline
\end{tabular}

The questionnaire was sent to member agencies by the INAHTA secretariat in November 2010, and followed up by three reminders. Responses were compared with those for the 2005 survey. Comparisons between the surveys for each of the questions were presented as the proportions of positive answers received.

\section{RESULTS}

Completed survey forms returned by the following agencies: AHTA, ASERNIP-S (Australia), LBI - HTA, GÖG (Austria), DECIT (Brazil), CADTH, IHE (Canada), DACEHTA, MIDT (Denmark), Finohta (Finland), HAS (France), DAHTA, GBA (Germany), HIQA (Ireland), ICTAHC (Israel), UVT (Italy), CNHTA (Korea), VASPVT (Lithuania), CENETEC (Mexico), CVZ (The Netherlands), MaHTAS (Malaysia), NOKC (Norway), AHTAPol (Poland), AETS, AVALIA-T, CAHIAQ, OSTEBA (Spain), SBU (Sweden), CDE (Taiwan), HITAP (Thailand), and HIS, NETSCC, NHSC (UK).

There were differences between the two surveys in the profile of responses, due to changes in the INAHTA membership. In 2010, there were responses from thirty-three members (63 percent response rate), compared with thirty-seven ( 90 percent response rate) in 2005. Nineteen agencies (58 percent) participated in both surveys, and fourteen had joined INAHTA since 2005. Distributions of responses by region are shown in Table 1.

Twenty-two agencies ( 67 percent) indicated that consumers were involved in some aspects of their HTA programs, compared with twenty-one (57 percent) in 2005. Of those agencies that do not currently involve consumers, 64 percent intended to do so in the future, the same proportion as in 2005 . Four agencies provided comments on use of consumers in the preparation of clinical practice guidelines, rather than with HTA.

Three agencies noted difficulties with involving consumers in their programs and provided the following comments.

"The horizon scanning technology briefings we produce do not lend themselves to the addition of consumer opinions. Consumers are represented on the panels that consider the technology briefings and prioritise them for technology appraisal. The subsequent technology appraisal also includes consideration of consumers and the production of an "easy-read/ understandable" version." 
Table 2. Approaches Used by Those Agencies That Involve Consumers in the Formulation of Assessment Topics

\begin{tabular}{lcc}
\hline Type of approach & $2010, \%$ & $2005, \%$ \\
\hline Consider suggestions made by consumers, including loosely-specified health technology topics & 83 & 100 \\
Use consumer input to the prioritizing process & 67 & 57 \\
Seek comment from consumers in refining the scope and nature of HTA projects & 54 & 43 \\
More detailed involvement in development of the HTA protocol, e.g. participation in committees & 58 & 43 \\
\hline
\end{tabular}

Table 3. Involvement of Consumers in Preparation of Assessments

Approach used

$2010, \%$

$2005, \%$

Consumers contacted to provide information or opinion on the technology being assessed

Consumers used to provide input to analysis/ interpretation of data or to drafting of some sections of HTA reports

Consumers used to review protocols and/or drafts of HTA reports
$86 \%$ (Sometimes 67\%, routinely: 33\%)
$59 \%$ (Sometimes 62\%, routinely: 38\%)
67\% (Sometimes 79\%, routinely: 21\%)
10\% (Sometimes: 100\%)
59\% (Sometimes 67\%, routinely: 33\%)
57\% (Sometimes: 60\%, routinely: 40\%)

"We consider involving consumers important and relevant, but also time consuming."

"Patient involvement is not a routine activity, this is intended only to our major projects. Among barriers to expanding patients' involvement there are time and resources constraints; difficulties in identifying appropriate consumers."

\section{Responses from Those Agencies That Involved Consumers}

In both surveys, 95 percent of agencies that involved consumers reported contact between patient organizations and their programs, and 45 percent also involved individual consumers.

Invitation from the agency remained the most frequently used method of bringing consumers into contact with the HTA process (90 percent). Compared with the 2005 survey, fewer agencies accepted requests from consumers on specific topics (59 percent versus 70 percent), while contact with the HTA process in response to publicity on forthcoming assessments was more common (45 percent versus 25 percent). Only a small proportion of agencies provide a training process for consumers who are involved in HTA programs (23 percent in 2010, 19 percent in 2005).

As before, a minority of agencies indicated that they avoided consumer involvement in some types of assessment (24 percent in 2005, 23 percent in 2010). Examples of where this occurred, provided by five agencies, included commercial in confidence evaluation of pharmaceuticals, assessments done on behalf of some other organizations, rapid assessments, and assessments in which there is no added value or benefit from the involvement of consumers. Horizon scanning and rapid assessments had been identified in the earlier survey as areas where consumers were not involved.

In 2010, 55 percent of agencies involved consumers in the formulation of assessment topics (67 percent in 2005). One agency does not generally seek consumers' input but involves them in a few projects regarding the assessment of treatments for psychiatric diagnoses. Approaches used by agencies are shown in Table 2. In the 2010 survey the proportion of agencies that considered consumers' suggestions was lower than in 2005, while that for each of the other (more detailed) approaches was higher.

Responses on involvement of consumers in the preparation of HTA reports are summarized in Table 3. In 2010, higher proportions of agencies contacted consumers or used them for interpretation of data and in drafting reports. Similar proportions in each survey used consumers to review protocols or reports. Routine involvement was higher in the 2010 survey for the first two types of task and lower for the third.

In 2010, 19 percent of agencies that involved consumers in their programs had undertaken appraisal of such involvement, compared with 29 percent in 2005 . Of the agencies that provided responses in 2010, one recorded numbers of reports where there has been consumer input, three noted the type of input, and three considered the influence of consumer input on product quality and relevance. None of them had considered the potential for conflicts of interest to influence consumer opinions.

\section{Preparation of Summaries for Consumers}

All agencies were asked whether they prepared summaries or versions of HTA reports that are intended to be easily understood by consumers. In the 2010 survey, 59 percent of agencies reported providing such material compared with 49 percent in 2005. Most of those that provided summaries of reports also involved consumers in their programs (84 percent in 2010 and 71 percent in 2005). Proportions of agencies that provided different types of summary are shown in Table 4. 
Table 4. Types of HTA Summary Prepared for Consumers

\begin{tabular}{lcc}
\hline Type of summary & $2010, \%$ & $2005, \%$ \\
\hline Information in newsletters or similar format & 47 & 65 \\
Brief one/two page summaries & 53 & 76 \\
More detailed consumer summaries & 39 & 24 \\
'Consumer versions' of HTA reports & 26 & 24 \\
\hline
\end{tabular}

\section{Involvement of Consumers in HTA Dissemination}

In the 2010 survey, 42 percent of agencies involved consumer organizations and/or individual consumers in the dissemination of HTA materials, compared with 33 percent in 2005.

\section{Consumer Perspectives in Assessments}

In response to the additional questions in the 2010 survey, seventeen ( 52 percent) of the agencies advised that their HTA reports consider consumer-reported attitudes and preferences, and thirteen ( 39 percent) that their reports include a section that assesses patient/consumer perspectives.

\section{Comparison of Agency Responses Over Time}

The 2010 responses from the sub-group of nineteen agencies that participated in both surveys were similar to those they provided in 2005. When compared with the total responses, The proportion of the nineteen agencies that involved consumers was similar to that for all agencies in 2010 and higher than the total for 2005 ( 65 percent versus 67 percent versus 57 percent). The proportion of sub-group agencies that prepared summaries for consumers was of the same order as for 2010 and higher than 2005 (63 percent versus 59 percent versus 49 percent). For those agencies that involved consumers, responses for all three groups were almost identical for involvement of patient organizations and methods of contact, involvement in topic formulation and in review of protocols and draft reports. The proportion of subgroup agencies that involve individual consumers was somewhat higher than the totals for both surveys ( 58 percent versus 46 percent versus 48 percent).

Responses from agencies that participated in only one survey were also compared. The proportion of the fourteen agencies from the 2010 survey that involved consumers was higher than for the eighteen that participated only in 2005 (71 percent versus 50 percent). The proportion of agencies that prepared summaries for consumers was also higher in 2010 than 2005 (50 percent versus 33 percent). For those agencies that involved consumers, responses for both groups were almost identical for involvement of patient organizations and of individual consumers, and methods of contact, involvement in topic formulation and in review of protocols and draft reports.

\section{DISCUSSION}

As in the previous survey, reported involvement of consumers in HTA varied among INAHTA members, reflecting differences in responsibilities and administrative arrangements. Comparison of findings from the two surveys is tentative, given the differences in response profiles, but there appear to be indications of increasing involvement in some activities.

A limitation in this comparison is the lower response rate by INAHTA members in the 2010 survey. The reasons for this decreased response are unclear. Possibly it was associated with increases in the workload and commitments of some member agencies. INAHTA projects rely heavily on the input from individual agencies and there are clearly limits to the time and resources that members can offer (14).

There were also differences between the surveys in the profile of responding agencies due to changes in INAHTA membership. Review of responses from agencies that participated in both surveys and those from single survey participants gave some indication of similarities between the two survey populations, particularly for those agencies that involved consumers. A feature of the 2010 survey was the increase in the proportion of agencies from Asia. It is considered unlikely that this change would have had any substantial effect on the comparison of surveys. The Asian agencies have been influenced by the well established HTA programs in Europe and have adopted the most suitable values for their own settings. Attitudes toward consumer involvement have been guided by these influences.

Of the agencies that provided responses, 67 percent involve consumers in aspects of their HTA programs, although not always routinely, a higher proportion than that in 2005. As in 2005, a large majority of agencies intend to involve consumers in the future. Similar findings were reported on types of consumer and how they are brought into contact with the HTA process. A majority of agencies accept requests on specific topics.

As in 2005, a minority of agencies avoid involving consumers in some types of assessment. Comments from these agencies point to practicalities in their programs that contribute to avoidance of contact. Time and resource constraints, requirements of rapid and horizon scanning assessments and projects undertaken in confidence all present challenges for wider patient involvement.

A much higher proportion of agencies in 2010 used consumer input to data analysis or drafting sections of HTA reports. A small majority in each survey used consumers to review of protocols or reports. Evaluation of consumer input to HTA programs remains uncommon.

In 2010, there was an increase in the proportion of agencies that provide summaries of HTA reports for consumers, and also in the proportion that involve consumers in dissemination. The additional items included in the 2010 survey indicated limited inclusion of consumer perspectives in assessment reports. 
Overall, the 2010 survey suggests that there is a trend to increased involvement of consumers by INAHTA members in their programs, continuing the generally positive response found in the earlier survey.

There appear to be similar issues to those for HTA in the involvement of consumers in the development of guidelines. A systematic review of patient and public involvement programs (PPIPs) in developing clinical practice guidelines found that of seventy-one descriptive reports twenty-three reported using PPIPs to “incorporate patients' values, preferences, knowledge, or perspectives in CPG recommendations" (15). Eccles et al. note that of the seventy-one reports only twenty-eight were dealing with consumers within the guideline development group itself and only twenty-nine were dealing with consumers involved in crafting recommendations (16). Barriers to consumer involvement that were identified resemble those that have emerged for health research and HTA. They included the discrepancy between the views of patients and experts, challenges of recruitment, obtaining representative input, consumers' lack of familiarity with technical issues and the degree of work/time involved. Eccles et al. comment that there are still few analytical empirical accounts of attempts to involve consumers in guideline development, and no robust evaluations of the effectiveness of different methods.

\section{POLICY IMPLICATIONS}

The involvement of consumers by many INAHTA agencies offers the potential to broaden the perspective of assessments and of the advice provided to decision makers. It also gives stakeholders further opportunity to gain information on health technologies that may help their own inputs to the policy process. There seems to be a trend toward increased involvement, but there are also barriers to wider routine participation of consumers in the HTA processes of these agencies.

\section{CONTACT INFORMATION}

David Hailey, MSc, PhD, Grad RIC, Professorial Fellow, University of Wollongong, Australia (dhailey@ozemail.com.au), Sophie Werkö, PhD. Swedish Council on Health Technology Assessment, Stockholm, Sweden, Rugayah Bakri M.B.B.CH, MPH, Ministry of Health Malaysia, Putrajaya, Malaysia, Alun Cameron, BSc (Hons), PhD. Australian Safety and Efficacy Register of New Interventional Procedures -Surgical, Adelaide, Australia, Britta Göhlen PhD, German Institute for Medical Documentation and Information, Cologne, Germany, Susan Myles BA (Hons), MSc, PhD, Healthcare Improvement Scotland, Glasgow, UK, Jasmine Pwu, PhD, Center for Drug Evaluation, Taipei, Taiwan, Jomkwan Yothasamut, BA, MA,
Health Intervention and Technology Assessment Program, Ministry of Public Health, Nonthaburi, Thailand

\section{CONFLICTS OF INTEREST}

The authors report no potential conflicts of interest.

\section{REFERENCES}

1. Hailey D. Consumer involvement in health technology assessment. HTA Initiative \#21. Edmonton: Alberta Heritage Foundation for Medical Research; 2005. [cited 2005 December 10]. Available from: http://www. ihe.ca/documents/HTA-FR21.pdf.

2. Pivik J, Rode E, Ward C. A consumer involvement model for health technology assessment in Canada. Health Policy. 2004;69:25368.

3. Ghersi D. Making it happen: approaches to involving consumers in Cochrane reviews. Eval Health Prof. 2002;25:270-83.

4. Sleath B, Rucker TD. Consumer participation in health policy decisions: empowerment or puffery? J Health Care Poor Underserved. 2001;12:3549.

5. Rowe R, Shepherd M. Public participation in the new NHS: no closer to citizen control? Soc Polit Adm. 2002;36:275.

6. Telford R, Boote JD, Cooper CL. What does it mean to involve consumers successfully in NHS research? A consensus study. Health Expect. 2004;7:209-20.

7. Royle J, Oliver S. Consumer involvement in the health technology assessment program. Int J Technol Assess Health Care. 2004;20:493-7.

8. Hailey D, Nordwall M. Survey on the involvement of consumers in health technology assessment programs. Int J Technol Assess Health Care. 2006;22:497-9.

9. Facey K, Boivin A, Gracia J, et al. Patients' perspectives in health technology assessment: a route to robust evidence and fair deliberation. Int $J$ Technol Assess Health Care. 2010;26:334-40.

10. Lee A, Skött LS, Hansen HP. Organizational and patient-related assessments in HTAs: state of the art. Int J Technol Assess Health Care. 2009;25:530-6.

11. Gagnon M-P, Desmartis M, Lepage-Savary D, et al. Introducing patients' and the public's perspectives to health technology assessment: A systematic review of international experiences. Int J Technol Assess Health Care 2011;27:31-42.

12. Gauvin FP, Abelson J, Giacomini M, Eyles J, Lavis JN. Moving cautiously: public involvement and the health technology assessment community. Int J Technol Assess Health Care. 2011;27:43-9.

13. Menon D, Stafinski T. Role of patient and public participation in health technology assessment and coverage decisions. Expert Rev Pharmacoecon Outcomes Res. 2011;11:75-89.

14. Hailey D. Development of the International Network of Agencies for Health Technology Assessment. Int J Technol Assess Health Care. 2009;25(Suppl 1):24-7.

15. Légaré F, Boivin A, van der Weijden T, et al. Patient and public involvement in clinical practice guidelines: a knowledge synthesis of existing programs. Med Decis Making. 2011;31:E45-74.

16. Eccles MP, Grimshaw JM, Shekelle P, Schünemann HJ, Woolf S. Developing clinical practice guidelines: target audiences, identifying topics for guidelines, guideline group composition and functioning and conflicts of interest. Implement Sci. 2012;7:60. 